\title{
Epidemiology and microbiology of nosocomial bloodstream infections: analysis of 482 cases from a retrospective surveillance study*
}

\author{
Jian-nong $\mathrm{WU}^{\S 1}$, Tie-er GAN ${ }^{\S 1}$, Yue-xian $\mathrm{ZHU}^{1}$, Jun-min $\mathrm{CAO}^{2}$, Cong-hua $\mathrm{JI}^{3}$, Yi-hua $\mathrm{WU}^{4}$, Bin $\mathrm{LV}^{\dagger \ddagger 5}$ \\ ( ${ }^{1}$ Department of Hospital Infection Control, the First Affiliated Hospital of Zhejiang Chinese Medical University, Hangzhou 310006, China) \\ $\left({ }^{2}\right.$ Microbiology Laboratory, the First Affiliated Hospital of Zhejiang Chinese Medical University, Hangzhou 310001, China) \\ $\left({ }^{3}\right.$ Clinical Evaluation and Analysis Center, the First Affiliated Hospital of Zhejiang Chinese Medical University, Hangzhou 310001, China) \\ ( ${ }^{4}$ Department of Epidemiology and Health Statistics, School of Public Health, Zhejiang University, Hangzhou 310058, China) \\ $\left({ }^{5}\right.$ Department of Gastroenterology, the First Affiliated Hospital of Zhejiang Chinese Medical University, Hangzhou 310001, China) \\ †E-mail: lvbin@medmail.com.cn \\ Received Apr. 18, 2014; Revision accepted Oct. 28, 2014; Crosschecked Dec. 24, 2014
}

\begin{abstract}
In many traditional Chinese medicine (TCM) hospitals, most patients are elderly with chronic diseases. Nosocomial bloodstream infections (nBSIs) are an important cause of morbidity and mortality. A retrospective surveillance study was performed to examine the epidemiology and microbiology of nBSIs in a TCM hospital from 2009 to 2011. A total of 482 patients with nBSIs were included in the study period. The incidence rate was $5.7 / 1000$ admissions. Escherichia coli $(25.5 \%)$ was the most common Gram-negative and coagulase-negative staphylococcus (CoNS) (14.1\%) was the most common Gram-positive organism isolated. One-third of the E. coli and Klebsiella pneumoniae isolated from the nBSIs were the third-generation cephalosporin-resistant. Half of the Acinetobacter species isolates were resistant to imipenem. Of all the CoNS isolates, $90.7 \%$ were resistant to methicillin. Carbapenems and glycopeptide were the most frequently used for nBSI therapy. Only about one-third of patients (157/482) received appropriate empirical therapy. Septic shock, hemodialysis, Pitt bacteremia score $>4$, urinary tract infection, and appropriate empirical therapy were most strongly associated with 28-d mortality. The incidence of nBSls was low in the TCM hospital but the proportion of nBSIs due to antibiotic-resistant organisms was high. A high Pitt bacteremia score was one of the most important risk factors for mortality in nBSIs. Therefore, the implementation of appropriate empirical therapy is crucial to improve the clinical outcome of nBSIs.
\end{abstract}

Key words: Nosocomial bloodstream infection, Traditional Chinese medicine hospital, Epidemiology, Microbiology doi: $10.1631 /$ jzus.B1400108 Document code: A CLC number: R181.2 2

\section{Introduction}

Between 575000 to 677000 episodes of bloodstream infections (BSIs) per year have been reported in North America as the cause of 79000 to 94000

\footnotetext{
¿ Corresponding author

$\S$ The two authors contributed equally to this work

* Project supported by the Projects of Zhejiang Province Non-profit Technology Research (No. 2013C33180), China

(17) ORCID: Jian-nong WU, http://orcid.org/0000-0001-8867-9860

(C) Zhejiang University and Springer-Verlag Berlin Heidelberg 2015
}

deaths annually. Reports from Europe showed that there were over 1.2 million episodes of BSIs with 157000 deaths per year (Goto and Al-Hasan, 2013). It is difficult to obtain precise estimates of nosocomial bloodstream infections (nBSIs) owing to the limited number of studies from nosocomial infection surveillance systems. Several population-based studies reported that $19.1 \%, 28 \%$, and $35 \%$ of the total episodes of BSI were nosocomial in the USA (Uslan et al., 2007), Canada (Lenz et al., 2012), and Finland (Skogberg et al., 2012), respectively, since the 2000s. Patients who developed nBSIs suffered high morbidity 
and mortality risks and had to bear heavy financial costs (Mittmann et al., 2012; Kaye et al., 2014). Many changes have recently occurred in the epidemiology of BSIs, particularly due to the emergence of drug-resistant organisms, which has significantly increased the treatment-failure rate and the risk of adverse outcomes (Lambert et al., 2011). In China, nBSIs accounted for one-third of nosocomial infections, followed by lower respiratory tract and urinary tract infections in the intensive care unit (ICU) (Ding et al., 2009).

Traditional Chinese medicine (TCM) is very popular in China. According to the Health Statistics book of 2012, a total of 2831 TCM hospitals accounted for $12.88 \%$ of the hospitals in China (2831/21979) (National Health and Family Planning Commission of the People's Republic of China, 2012). Patients with chronic diseases, including hypertension, diabetes, cancer, hematological malignancies, and other illnesses, often chose TCM hospitals for their treatment. Furthermore, post-operative chemotherapy of malignant tumors, diabetes, and chronic obstructive pulmonary disease patients continued to increase in our hospital for nearly a decade (Jiang et al., 2007). These patients often had a long hospital stay, were elderly, and had underlying diseases, which contributed to a higher risk of suffering from nBSIs. However, studies are limited on nBSIs in TCM hospitals and the epidemiology and microbiology of nBSIs are still unclear in TCM hospitals. Therefore, a retrospective survey was performed to evaluate the epidemiological features of nBSIs in a tertiary TCM hospital, in order to describe the characteristics of the species distribution and to identify the factors influencing mortality.

\section{Materials and methods}

\subsection{Study design and data collection}

We undertook a retrospective surveillance study of nBSIs in the First Affiliated Hospital of Zhejiang Chinese Medical University in China, from January 2009 to December 2011. The First Affiliated Hospital of Zhejiang Chinese Medical University is a 1200 bed TCM hospital. The nBSI was diagnosed if one or more cultures of the sampled blood yielded a pathogenic organism, at least $48 \mathrm{~h}$ after admission. If the bloodstream isolate was a potential skin contaminant (e.g., coagulase-negative staphylococcus (CoNS), Corynebacterium, Bacillus, or Propionibacterium), two or more separate blood culture sets were required for the diagnosis (Weinstein et al., 1983).

The data were obtained from the electronic medical records without any additional tests performed for the purpose of this study. The data collected for each patient included demographic information, predisposing clinical conditions, underlying diseases, microbiological data, as well as the antimicrobial treatment information and the susceptibilities of the causative pathogens.

\subsection{Variables and definitions}

The main clinical outcome was measured by a 28-d mortality rate. Predisposing clinical conditions included diabetes, neutropenia, septic shock, chemotherapy, parenteral nutrition, hemodialysis, and the usage of invasive devices. Based on the standard definition, septic shock is a medical condition that occurs as a result of severe infection and sepsis (Bone et al., 1992). Underlying diseases included malignancies, neurological dysfunctions, cardiovascular malfunctions, gastroenterological diseases, respiratory dysfunctions, and renal diseases. The acute severity of the illness was retrospectively assessed on the day before the diagnosis of nBSIs using the Pitt bacteremia score. This is a validated index, based on mental status, need for ventilation and vital signs, which has been used in previous studies (Hilf et al., 1989; Lim et al., 2014). Source of infection was determined from clinical and microbiological data and using CDC/NHSN criteria (Horan et al., 2008).

Detailed information was recorded on the antimicrobial drug treatments during the period from $3 \mathrm{~d}$ prior to the collection of the first positive blood culture up to $7 \mathrm{~d}$ after it. Empirical antibiotic therapy was considered appropriate when an antibiotic, that had in vitro activity against the causative organism, was given during the first $48 \mathrm{~h}$. If the isolates were resistant or if the dose or the route of administration was insufficient, treatment was considered to be inappropriate (Pedersen et al., 2003; Schonheyder and Sogaard, 2010; Lim et al., 2014).

The Ethics Committee of our hospital approved the procedures, and the data collected from this study were kept confidential. 


\subsection{Microbiological test}

One or double blood specimens were collected from each patient at different sites within $5 \mathrm{~min}$. The identification of blood isolates was carried out according to routine methods by the staff of the microbiology laboratory in our hospital. Antibiotic susceptibility testing was performed using the modified broth microdilution method. Minimum inhibitory concentration (MIC) breakpoints and quality control protocols were used according to the standards established by the Clinical and Laboratory Standards Institute (2007). Antibiotic susceptibility data was analyzed with WHONET 5.6 software.

\subsection{Statistical analysis}

The results were expressed as the mean \pm standard deviation (SD) for continuous variables, or as a proportion of the total number of patients or isolates for categorical variables. Univariate analysis was used to examine the association between predictor variables and the risk of mortality at $28 \mathrm{~d}$. Variables that were statistically significant in the univariate analysis were then included in a multiple logistic regression analysis to eliminate potential confounding factors. For source of infection, central line associated blood stream infection (CLABSI) was used as the baseline category when performing regression analysis, as in the previous study of Melzer and Welch (2013). This was chosen because CLABSIs are well defined and are associated with a large number of BSIs. $P$ values of $<0.05$ were considered to be statistically significant The SPSS 16.0 for Windows software package (Version 16.0) was used for this analysis.

\section{Results}

\subsection{Study population and patient characteristics}

During the three-year study period, there were 482 significant $\mathrm{nBSI}$ episodes. The incidence rate of nBSIs was 5.7/1000 hospital admissions. Patient characteristics and distribution of nBSIs are shown in Table 1.

\subsection{Microbiological features}

The poly-microbial accounted for 27 out of 482 episodes $(5.6 \%)$. Of all the nBSI isolates,
Table 1 Patient characteristics of the 482 patients with nBSIs

\begin{tabular}{lc}
\hline \multicolumn{1}{c}{ Parameter } & Value $^{*}$ \\
\hline Patient demographics & $62.96 \pm 21.4$ \\
Age (year) & $267(55.4 \%)$ \\
Male & \\
nBSI distribution & $127(26.3 \%)$ \\
ICU & $108(22.4 \%)$ \\
Hematology & $80(16.6 \%)$ \\
Oncology & $167(34.6 \%)$ \\
Others & \\
Underlying disease & $124(25.7 \%)$ \\
Solid tumor & $104(21.6 \%)$ \\
Hematological malignancy & $53(11.0 \%)$ \\
Gastroenterology & $43(8.9 \%)$ \\
Neurologic & $36(7.5 \%)$ \\
Respiratory & $29(6.0 \%)$ \\
Cardiovascular & $16(3.3 \%)$ \\
Renal &
\end{tabular}

"Values are expressed as number of patients (percentage), except age (mean $\pm \mathrm{SD})$

Gram-negative organisms were the cause of approximately three-fifths $(61.8 \%)$; Gram-positive organisms accounted for $33.6 \%$; and, fungi accounted for only $4.6 \%$. Escherichia coli $(25.5 \%)$ was the principal organism responsible for nBSIs, followed by CoNS (14.1\%) and Klebsiella pneumoniae (11.2\%). When analyzed by the clinical department, the following patterns emerged: E. coli, K. pneumoniae, and $S$. aureus were the top three isolated pathogens for the general wards, except for the ICU, where CoNS (29, $22.8 \%$ ) and Acinetobacter spp. (17, 13.4\%) were more frequently isolated (Table 2). 
Table 2 Distribution of the most frequently isolated pathogens causing nBSIs and number of deaths caused by nBSIs in different clinical departments

\begin{tabular}{lccccc}
\hline \multirow{2}{*}{ Pathogen } & \multicolumn{5}{c}{ No. of patients (No. of deaths) } \\
\cline { 2 - 5 } & ICU $(n=127)$ & Hematology $(n=108)$ & Oncology $(n=80)$ & Others $(n=167)$ & Total $(n=482)$ \\
\hline CoNS & $29(7)$ & $8(1)$ & $6(0)$ & $25(0)$ & $68(8)$ \\
E. coli & $13(2)$ & $29(5)$ & $30(4)$ & $51(2)$ & $123(13)$ \\
A. baumannii & $17(14)$ & $2(2)$ & $0(0)$ & $3(1)$ & $22(17)$ \\
S. aureus & $16(3)$ & $8(1)$ & $11(0)$ & $18(1)$ & $53(5)$ \\
K. pneumoniae & $14(3)$ & $16(7)$ & $8(3)$ & $16(2)$ & $54(15)$ \\
P. aeruginosa & $3(1)$ & $13(4)$ & $1(0)$ & $4(2)$ & $21(7)$ \\
E. faecium & $8(4)$ & $7(3)$ & $4(0)$ & $5(1)$ & $24(8)$ \\
\hline
\end{tabular}

"Number of the most common pathogens associated with death in nBSI patients

\subsection{Antimicrobial susceptibility}

Antimicrobial resistance levels for the most common organisms causing nBSIs are shown in Tables 3 and 4 . Methicillin resistance was detected in $60.0 \%$ of the 30 S. aureus and $90.7 \%$ of the 54 CoNS isolates (Table 3). Resistance to the third-generation cephalosporins was found in $38.6 \%$ of the 105 E. coli and $35.5 \%$ of the $31 \mathrm{~K}$. pneumoniae isolates in nBSI patients (Table 4). Extended-spectrum $\beta$-lactamase (ESBL)-positive E. coli and $K$. pneumoniae accounted for $79.8 \%$ and $57.1 \%$ of isolates, respectively. Imipenem resistance was only detected in $6.1 \%$ of the 114 E. coli and $6.5 \%$ of the $31 \mathrm{~K}$. pneumoniae isolates. However, resistance to imipenem was observed in $50.0 \%$ of Acinetobacter spp. isolates.

\subsection{Antimicrobial drugs}

A total of 479 patients (99.4\%) used antibiotics during the period from $3 \mathrm{~d}$ before the collection of the first positive blood culture to $7 \mathrm{~d}$ after it. Of those, $85.2 \%$ used antibiotics before blood specimen collection. Only about one-third of patients (157/482) received appropriate empirical therapy (Table 1). Carbapenems were the antimicrobial drugs that were the most frequently given $(n=208 ; 43.2 \%)$, followed by glycopeptides $(n=128 ; 26.6 \%)$ and cefoperazonesulbactam $(n=123 ; 25.5 \%)$, as shown in Fig. 1 .

\subsection{Clinical outcomes and risk factors for the 28-d mortality}

The overall 28-d mortality rate in all the patients with nBSIs was $16.8 \%(81 / 482)$. The top three pathogens associated with the fatalities included Acinetobacter spp., Enterococcus faecium, and Pseudomonas aeruginosa, with mortality rates of $77.3 \%$ (17/22), 33.3\% (8/24), and 29.2\% (7/24), respectively.
Table 3 Rates of antimicrobial resistance among Gram-positive bacteria most frequently isolated from patients with nBSIs

\begin{tabular}{lccc}
\hline \multirow{2}{*}{$\begin{array}{c}\text { Antimicrobial } \\
\text { drug }\end{array}$} & \multicolumn{3}{c}{$n_{\mathrm{ri}} / n_{\mathrm{it}}(\%$ resistant $)$} \\
\cline { 2 - 4 } & $\begin{array}{c}\text { S. aureus } \\
(n=53)\end{array}$ & $\begin{array}{c}\text { CoNS } \\
(n=68)\end{array}$ & $\begin{array}{c}\text { Enterococcus } \\
(n=35)\end{array}$ \\
\hline Ampicillin & & & $12 / 18$ \\
& & & $(66.7 \%)$ \\
Ampicillin- & $8 / 19$ & $16 / 28$ & \\
sulbactam & $(42.1 \%)$ & $(57.1 \%)$ & \\
Cefazolin & $9 / 18$ & $17 / 28$ & \\
& $(50.0 \%)$ & $(60.7 \%)$ & \\
Ciprofloxacin & $12 / 22$ & $25 / 34$ & \\
& $(54.5 \%)$ & $(73.5 \%)$ & \\
Clindamycin & $12 / 35$ & $22 / 57$ & \\
& $(34.3 \%)$ & $(38.6 \%)$ & \\
Co-trimoxazole & $7 / 25$ & $14 / 44$ & \\
& $(28.0 \%)$ & $(31.8 \%)$ & \\
Erythromycin & $20 / 35$ & $47 / 57$ & $25 / 28$ \\
& $(57.1 \%)$ & $(82.5 \%)$ & $(89.3 \%)$ \\
Gentamicin & $16 / 37$ & $34 / 61$ & \\
& $(43.2 \%)$ & $(55.7 \%)$ & \\
Levofloxacin & $24 / 34$ & $43 / 59$ & $22 / 30$ \\
& $(70.6 \%)$ & $(72.9 \%)$ & $(73.3 \%)$ \\
Linezolid & $3 / 35$ & $4 / 58$ & $1 / 28$ \\
& $(8.57 \%)$ & $(6.9 \%)$ & $(3.6 \%)$ \\
Methicillin & $18 / 30$ & $49 / 54$ & \\
& $(60.0 \%)$ & $(90.7 \%)$ & \\
Nitrofurantoin & $1 / 38$ & $2 / 61$ & $5 / 27$ \\
& $(2.6 \%)$ & $(3.3 \%)$ & $(18.5 \%)$ \\
Penicillin & $35 / 35$ & $56 / 57$ & $8 / 26$ \\
& $(100)$ & $(98.2 \%)$ & $(30.8 \%)$ \\
Tetracycline & $3 / 23$ & $8 / 19$ & $12 / 28$ \\
& $(13.0 \%)$ & $(42.1 \%)$ & $(42.9 \%)$ \\
Vancomycin & $0 / 35$ & $0 / 60$ & $0 / 28$ \\
& $(0 \%)$ & $(0 \%)$ & $(0 \%)$ \\
\hline
\end{tabular}

$n_{\mathrm{ri}}$ : number of resistant isolates; $n_{\mathrm{it}}$ : number of isolates tested

Although the prevalence rates of CoNS and E. coli isolates were the highest in ICU and general wards respectively, the corresponding mortality was not the highest. The pathogen that was most frequently associated with fatality in the ICU was Acinetobacter spp. $(82.3 \%, 14 / 17)$, while in hematology and oncology it was K. pneumoniae $(43.8 \%, 7 / 16 ; 37.5 \%, 3 / 8)$ (Table 2). 
Table 4 Rates of antimicrobial resistance among Gram-negative bacteria most frequently isolated from patients with nBSIs

\begin{tabular}{lccc}
\hline \multicolumn{1}{c}{ Antimicrobial } & \multicolumn{3}{c}{$n_{\mathrm{ri}} / n_{\mathrm{it}}(\%$ resistant $)$} \\
\cline { 2 - 4 } drug & E. coli $(n=123)$ & Acinetobacter spp. $(n=22)$ & K. pneumonia $(n=54)$ \\
\hline Ampicillin-sulbactam & $78 / 103(75.7 \%)$ & $1 / 9(11.1 \%)$ & $22 / 32(68.8 \%)$ \\
Amikacin & $12 / 105(11.4 \%)$ & $7 / 9(77.8 \%)$ & $1 / 31(3.2 \%)$ \\
Aztreonam & $63 / 105(60.0 \%)$ & $6 / 9(66.7 \%)$ & $15 / 30(50.0 \%)$ \\
Cefepime & $21 / 105(20.0 \%)$ & $6 / 25(24.0 \%)$ & $7 / 31(22.6 \%)$ \\
Cefotetan & $30 / 96(31.3 \%)$ & $6 / 9(66.7 \%)$ & $10 / 31(32.3 \%)$ \\
Ceftazidime & $33 / 105(31.4 \%)$ & & $12 / 31(38.7 \%)$ \\
Ceftriaxone & $48 / 105(45.8 \%)$ & $7 / 11(63.6 \%)$ & $23 / 38(60.5 \%)$ \\
Ciprofloxacin & $46 / 102(45.1 \%)$ & $1 / 9(11.1 \%)$ & $16 / 31(51.6 \%)$ \\
Co-trimoxazole & $48 / 107(44.9 \%)$ & & $24 / 42(57.1 \%)$ \\
ESBL-positive & $87 / 109(79.8 \%)$ & $1 / 9(11.1 \%)$ & $16 / 44(36.4 \%)$ \\
Gentamicin & $49 / 107(45.8 \%)$ & $4 / 8(50.0 \%)$ & $2 / 31(6.5 \%)$ \\
Imipenem & $7 / 114(6.1 \%)$ & $10 / 17(58.8 \%)$ & $26 / 44(59.1 \%)$ \\
Levofloxacin & $53 / 110(48.2 \%)$ & &
\end{tabular}

$n_{\mathrm{ri}}$ : number of resistant isolates; $n_{\mathrm{it}}$ : number of isolates tested

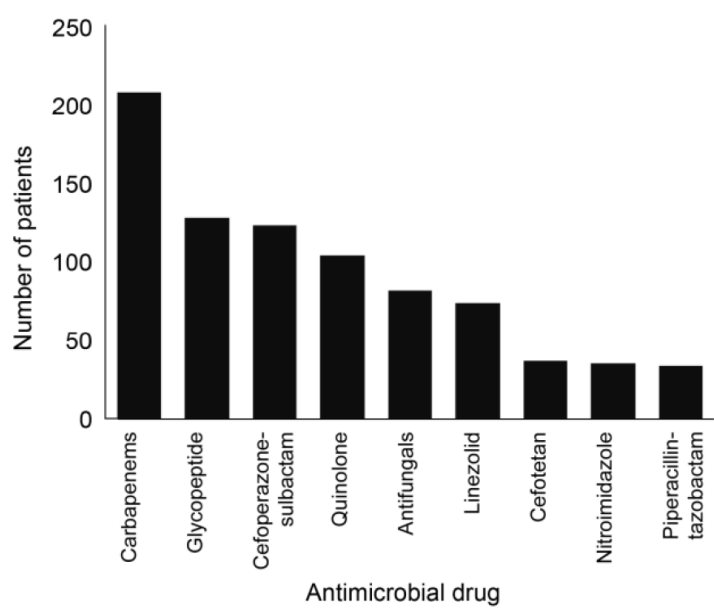

Fig. 1 Most frequent use of antimicrobial drugs The most frequent antimicrobial drug treatments during the period from $3 \mathrm{~d}$ prior to the collection of the first positive blood culture up to $7 \mathrm{~d}$ after it

Univariate analyses of 28 -d mortality are presented in Table 5. A number of variables were statistically significantly associated with 28 -d mortality, including septic shock, central venous catheter, urinary catheter, ventilator, hemodialysis, parenteral nutrition, appropriate empirical therapy, Pitt bacteremia score, and source of infection. The results of multiple logistic regression analysis are presented in Table 6. After adjustment for potential confounding factors, we found that septic shock (odds ratio $(\mathrm{OR})=$ 2.77, $P=0.01)$, hemodialysis $(\mathrm{OR}=3.29, P=0.012)$,
Pitt bacteremia score $>4(\mathrm{OR}=12.88, P<0.001)$, and urinary tract infection $(\mathrm{OR}=11.12, P<0.001)$ were strongly associated with 28-d mortality from nBSIs, while appropriate empirical therapy was a protective factor for 28-d mortality ( $\mathrm{OR}=0.23, P=0.001$ ).

\section{Discussion}

Hospital surveillance studies on nosocomial infections could be useful to discover specific issues related to species distribution and resistance patterns of the pathogens causing nBSIs. It is essential to evaluate the etiologic agents and clinical symptoms together to make sure that the surveillance data are reliable. Therefore, we performed a clinically oriented surveillance by collecting the data on the defined infections rather than solely evaluating the culture results in this study.

The incidence of nBSIs in this study was 5.7/1000 hospital admissions, which was not as high as expected and close to the incidence levels of the US with 6.0/1000 admissions (Wisplinghoff et al., 2004). Other studies reported that the incidence of nBSIs was 11.6 to 15.5 episodes per 1000 admissions in Thailand (Hortiwakul et al., 2012) and 16.0 to 31.2 episodes per 1000 admissions in Europe (Rodríguez-Créixems et al., 2008). The relatively low incidence rate in this study might be explained by following reasons. One 
Table 5 Univariate analysis of 28-d mortality in nBSIs

\begin{tabular}{|c|c|c|}
\hline Risk factor & $\begin{array}{l}\text { Adjusted odds ratio } \\
(95 \% \mathrm{CI})\end{array}$ & $P$ value \\
\hline Septic shock & $4.34(2.63-7.14)$ & $<0.001$ \\
\hline Central venous catheter & $3.19(1.42-7.17)$ & 0.005 \\
\hline Urinary catheter & $3.92(2.30-6.70)$ & $<0.001$ \\
\hline Ventilator & $1.87(1.13-3.10)$ & 0.015 \\
\hline Diabetes & $0.92(0.50-1.66)$ & 0.771 \\
\hline Surgery & $0.67(0.34-1.33)$ & 0.254 \\
\hline Hemodialysis & $3.07(1.70-5.56)$ & $<0.001$ \\
\hline Chemotherapy & $0.62(0.35-1.08)$ & 0.093 \\
\hline Neutropenia & $1.35(0.73-2.48)$ & 0.340 \\
\hline Parenteral nutrition & $2.08(1.27-3.40)$ & 0.004 \\
\hline $\begin{array}{l}\text { Appropriate empirical } \\
\text { therapy }\end{array}$ & $0.27(0.14-0.54)$ & $<0.001$ \\
\hline \multicolumn{3}{|l|}{ Pitt bacteremia score } \\
\hline $0-1$ & 1 & \\
\hline $2-4$ & $2.39(1.15-4.98)$ & 0.020 \\
\hline$>4$ & $14.14(7.55-26.46)$ & $<0.001$ \\
\hline \multicolumn{3}{|l|}{ Source of infection } \\
\hline Central venous catheter & 1 & \\
\hline Abdominal infection & $0.43(0.22-0.85)$ & 0.015 \\
\hline Urinary tract infection & $7.96(3.11-20.33)$ & $<0.001$ \\
\hline Pulmonary infection & $0.25(0.09-0.73)$ & 0.012 \\
\hline Others/unknown & $1.21(0.62-2.38)$ & 0.575 \\
\hline
\end{tabular}

Table 6 Multivariate logistic regression analysis of 28-d mortality in nBSIs

\begin{tabular}{lcr}
\hline \multicolumn{1}{c}{ Risk factor } & $\begin{array}{c}\text { Adjusted odds ratio } \\
(95 \% \text { CI })\end{array}$ & $P$ value \\
\hline Septic shock & $2.77(1.27-6.02)$ & 0.010 \\
Central venous catheter & $2.37(0.71-7.92)$ & 0.161 \\
Urinary catheter & $1.75(0.79-3.87)$ & 0.167 \\
Ventilator & $0.77(0.23-2.52)$ & 0.664 \\
Hemodialysis & $3.29(1.30-8.34)$ & 0.012 \\
Parenteral nutrition & $1.44(0.72-2.87)$ & 0.300 \\
Appropriate empirical & $0.23(0.10-0.56)$ & 0.001 \\
$\quad$ therapy & & \\
Pitt bacteremia score & & \\
0-1 & 1 & 0.261 \\
2-4 & $0.45(0.11-1.82)$ & \\
$>4$ & $12.88(5.58-29.70)$ & $<0.001$ \\
Source of infection & & 0.872 \\
Central venous catheter & 1 & $<0.001$ \\
Abdominal infection & $1.07(0.47-2.45)$ & 0.053 \\
Urinary tract infection & $11.12(2.97-41.71)$ & 0.090 \\
Pulmonary infection & $0.29(0.08-1.02)$ & \\
Others or unknown & $2.47(0.87-7.05)$ & \\
\hline
\end{tabular}

probable reason might be the low number and incorrect timing of blood cultures, which may have a significant effect on diagnostic of BSIs (Rampini et al., 2011; Schmitz et al., 2013). Another reason might be the small proportion of surgical and critically ill patients in our hospital. Many studies showed that these patients had a significantly higher incidence of $\mathrm{nBSIs}$ (Ding et al., 2009; Trethon et al., 2012). The incidence of nBSIs was up to $34.25 / 1000$ in the cardiac surgery ICUs (Trethon et al., 2012).

E. coli $(25.5 \%)$, CoNS (14.1\%), and K. pneumoniae $(11.2 \%)$ were the top three bacteria isolated in this study. The patterns of bacterial distribution in this study were consistent with those reported in a teaching hospital in Beijing (Lu et al., 2013) and the nationwide surveillance research of China (Wei et al., 2012). In the present study, antibiotic resistance within the group of Gram-negative bacteria was worrisome, especially in the case of Acinetobacter spp. with a rate as high as $50 \%$ for resistance to imipenem. Imipenemresistant Acinetobacter spp. mainly occurred in ICU. Since resistance to imipenem was usually associated with multidrug resistance, very few antibacterials remain potent (Ye et al., 2010). This could explain why patients with Acinetobacter spp. infection had the highest mortality rate $(82.3 \%, 14 / 17)$.

As for the use of antibiotics, almost every patient was treated with broad-spectrum antibiotics. About $85.2 \%$ of them used the antibiotics before blood specimen collection, which was much higher than the $20 \%$ reported by a multi-center study and included the 1100 ICU patients with nBSIs (Tabah et al., 2012). However, compared with that study, the most frequent use of antibiotics for treating BSIs was the same; they were carbapenems and glycopeptides. Moreover, only one-third of patients (157/482) received appropriate empirical therapy in our hospital, which is much lower than those in other hospitals (Townell et al., 2014).

In the present study, septic shock, hemodialysis, Pitt bacteremia score $>4$, and source from urinary tract infection were shown to be the most common risk factors for the 28-d mortality of nBSI. The Pitt bacteremia score is a marker of severity of illness in patients with nBSIs and patients with scores $>4$ are considered to be critically ill. In line with the results of previous studies (Neuner et al., 2011; Retamar et al., 2011; Melzer and Welch, 2013), in our hospital the Pitt bacteremia score was strongly correlated with 28-d mortality. Compared with CLABSI, we demonstrated a significant association between urinary tract 
source of BSI and death at $28 \mathrm{~d}$. This result has also been validated in a multi-center study in the UK (Melzer and Welch, 2013). However, appropriate empirical therapy can protect patients from mortality at $28 \mathrm{~d}$. The mortality rate was reduced by approximately 4-fold in patients receiving appropriate empirical therapy compared to those without $(\mathrm{OR}=0.23$, $P=0.001)$. As shown in other studies, appropriate empirical antibiotic treatment has been known to be the most effective treatment for BSIs (Sogaard et al., 2011) and inappropriate empirical antibiotic therapy has been one of the most important risk factors for mortality in nBSIs (Tabah et al., 2012). Therefore, how to improve the empirical use of antibiotics in the early period of BSIs in order to reduce the 28-d mortality in our hospital should be elaborated in future studies.

In conclusion, the incidence of nBSIs was low in the TCM hospital but the proportion of nBSIs due to antibiotic-resistant organisms requires more attention. Efficient control methods are needed to decrease antibiotic drug resistance and to ensure patients receive effective treatment. A high Pitt bacteremia score was identified as one of the most important risk factors for mortality in nBSIs. Adequate empirical therapy is independently associated with decreased mortality in patients with nBSIs. Therefore, programs to improve the quality of empirical therapy in patients with suspected nBSIs and optimization of definitive therapy should be implemented.

\section{Acknowledgements}

We would like to thank Tim ROSS (the overseas student of the First Affiliated Hospital of Zhejiang Chinese Medical University, China) and Micheal HOUGHTON (the chairman of ICNet International Ltd., UK) for their editing, helpful comments, and suggestions for this manuscript.

\section{Compliance with ethics guidelines}

Jian-nong WU, Tie-er GAN, Yue-xian ZHU, Jun-min CAO, Cong-hua JI, Yi-hua WU, and Bin LV declare that they have no conflict of interest.

This article does not contain any studies with human or animal subjects performed by any of the authors.

\section{References}

Bone, R.C., Balk, R.A., Cerra, F.B., et al., 1992. American College of Chest Physicians/Society of Critical Care Medicine Consensus Conference: definitions for sepsis and organ failure and guidelines for the use of innovative therapies in sepsis. Crit. Care Med., 20(6):864-874. [doi:10.1097/00003246-199206000-00025]

Clinical and Laboratory Standards Institute, 2007. Perfor- mance Standards for Antimicrobial Susceptibility Testing: 17th Informational Supplement. Vol. 27, No. 1, M100-S17, CLSI, Wayne, PA, USA.

Ding, J.G., Sun, Q.F., Li, K.C., et al., 2009. Retrospective analysis of nosocomial infections in the intensive care unit of a tertiary hospital in China during 2003 and 2007. BMC Infect. Dis., 9(1):115. [doi:10.1186/1471-2334-9115]

Goto, M., Al-Hasan, M.N., 2013. Overall burden of bloodstream infection and nosocomial bloodstream infection in North America and Europe. Clin. Microbiol. Infect., 19(6): 501-509. [doi:10.1111/1469-0691.12195]

Hilf, M., Yu, V.L., Sharp, J., et al., 1989. Antibiotic therapy for Pseudomonas aeruginosa bacteremia: outcome correlations in a prospective study of 200 patients. Am. J. Med., 87(5):540-546. [doi:10.1016/S0002-9343(89)80611-4]

Horan, T.C., Andrus, M., Dudeck, M.A., 2008. CDC/NHSN surveillance definition of health care-associated infection and criteria for specific types of infections in the acute care setting. Am. J. Infect. Control, 36(5):309-332. [doi:10.1016/j.ajic.2008.03.002]

Hortiwakul, T., Nagij, S., Chusri, S., et al., 2012. Nosocomial bloodstream infection in Songklanagarind Hospital: outcome and factors influencing prognosis. J. Med. Assoc. Thai., 95(2):170-174.

Jiang, W.D., Ji, C.H., Hu, Q.W., 2007. Dominant disease analysis of Traditional Chinese Medicine Hospital of Zhejiang Province. Zhejiang Stat., 25(6):24-25 (in Chinese).

Kaye, K.S., Marchaim, D., Chen, T.Y., et al., 2014. Effect of nosocomial bloodstream infections on mortality, length of stay, and hospital costs in older adults. J. Am. Geriatr. Soc., 62(2):306-311. [doi:10.1111/jgs.12634]

Lambert, M.L., Suetens, C., Savey, A., et al., 2011. Clinical outcomes of health-care-associated infections and antimicrobial resistance in patients admitted to European intensive-care units: a cohort study. Lancet Infect. Dis., 11(1):30-38. [doi:10.1016/S1473-3099(10)70258-9]

Lenz, R., Leal, J.R., Church, D.L., et al., 2012. The distinct category of healthcare associated bloodstream infections. BMC Infect. Dis., 12(1):85. [doi:10.1186/1471-2334-12-85]

Lim, C.J., Cheng, A.C., Kong, D.C., et al., 2014. Communityonset bloodstream infection with multidrug-resistant organisms: a matched case-control study. BMC Infect. Dis., 14(1):126. [doi:10.1186/1471-2334-14-126]

Lu, Y., Guo, P., Ye, Y.J., et al., 2013. Clinical and microbiological features of community-acquired and nosocomial bloodstream infections in the surgical department of a Tertiary-Care Hospital in Beijing. Chin. Med. J. (Engl.), 126(22):4242-4246.

Melzer, M., Welch, C., 2013. Outcomes in UK patients with hospital-acquired bacteraemia and the risk of catheterassociated urinary tract infections. Postgrad. Med. J., 89(1052):329-334. [doi:10.1136/postgradmedj-2012-131393]

Mittmann, N., Koo, M., Daneman, N., et al., 2012. The economic burden of patient safety targets in acute care: a systematic review. Drug Healthc. Patient Saf., 4:141-165. [doi:10.2147/DHPS.S33288]

National Health and Family Planning Commission of the People's Republic of China, 2012. 2012 China health statistics annual inspection. Available from http://www. 
nhfpc.gov.cn/htmlfiles/zwgkzt/ptjnj/year2012/index2012. html [Accessed on Aug. 21, 2013].

Neuner, E.A., Yeh, J.Y., Hall, G.S., et al., 2011. Treatment and outcomes in carbapenem-resistant Klebsiella pneumoniae bloodstream infections. Diagn. Microbiol. Infect. Dis., 69(4):357-362. [doi:10.1016/j.diagmicrobio.2010.10.013]

Pedersen, G., Schonheyder, H.C., Sorensen, H.T., 2003. Source of infection and other factors associated with case fatality in community-acquired bacteremia - a Danish population-based cohort study from 1992 to 1997. Clin. Microbiol. Infect., 9(8):793-802. [doi:10.1046/j.14690691.2003.00599.x]

Rampini, S.K., Bloemberg, G.V., Keller, P.M., et al., 2011. Broad-range 16S rRNA gene polymerase chain reaction for diagnosis of culture-negative bacterial infections. Clin. Infect. Dis., 53(12):1245-1251. [doi:10.1093/cid/cir692]

Retamar, P., Portillo, M.M., Lopez-Prieto, M.D., et al., 2011. Impact of inadequate empirical therapy on the mortality of patients with bloodstream infections: a propensity score-based analysis. Antimicrob. Agents Chemother., 56(1):472-478. [doi:10.1128/AAC.00462-11]

Rodríguez-Créixems, M., Alcala, L., Munoz, P., et al., 2008. Bloodstream infections: evolution and trends in the microbiology workload, incidence, and etiology, 1985-2006 Medicine (Baltimore), 87(4):234-249. [doi:10.1097/MD. 0b013e318182119b]

Schmitz, R.P., Keller, P.M., Baier, M., et al., 2013. Quality of blood culture testing - a survey in intensive care units and microbiological laboratories across four European countries. Crit. Care, 17(5):R248. [doi:10.1186/cc13074]

Schonheyder, H.C., Sogaard, M., 2010. Existing data sources for clinical epidemiology: the North Denmark Bacteremia Research Database. Clin. Epidemiol., 9(2):171-178. [doi:10. 2147/CLEP.S10139]

Skogberg, K., Lyytikainen, O., Ollgren, J., et al., 2012. Population-based burden of bloodstream infections in Finland. Clin. Microbiol. Infect., 18(6):E170-E176. [doi:10.1111/j.1469-0691.2012.03845.x]

Sogaard, M., Norgaard, M., Dethlefsen, C., et al., 2011. Temporal changes in the incidence and 30-day mortality associated with bacteremia in hospitalized patients from 1992 through 2006: a population-based cohort study. Clin Infect. Dis., 52(1):61-69. [doi:10.1093/cid/ciq069]

Tabah, A., Koulenti, D., Laupland, K., et al., 2012. Characteristics and determinants of outcome of hospital-acquired bloodstream infections in intensive care units: the EUROBACT international cohort study. Intensive Care Med., 38(12):1930-1945. [doi:10.1007/s00134-012-2695-9]

Townell, N., McDougall, D., Playford, E.G., 2014. Parenteral nutrition-associated bloodstream infection in an Australian teaching hospital - an 8-year retrospective study of over 11000 PN-days. Scand. J. Infect. Dis., 46(5): 361-367. [doi:10.3109/00365548.2014.880185]

Trethon, A., Prinz, G., Varga, A., et al., 2012. Characteristics of nosocomial bloodstream infections at a Hungarian cardiac surgery centre. Acta Microbiol. Immunol. Hung., 59(2):271-283. [doi:10.1556/AMicr.59.2012.2.12]

Uslan, D.Z., Crane, S.J., Steckelberg, J.M., et al., 2007. Ageand sex-associated trends in bloodstream infection: a population-based study in Olmsted County, Minnesota.
Arch. Intern. Med., 167(8):834-839. [doi:10.1001/archinte. 167.8.834]

Wei, Z.Q., Shen, P., Chen, Y.B., et al., 2012. Mohnarin report of 2010: bacterial composing and resistance in bloodstream infections. Chin. J. Nosocomiol., 22(3):465-470 (in Chinese).

Weinstein, M.P., Murphy, J.R., Reller, L.B., et al., 1983. The clinical significance of positive blood cultures: a comprehensive analysis of 500 episodes of bacteremia and fungemia in adults. II. Clinical observations, with special reference to factors influencing prognosis. Clin. Infect. Dis., 5(1):54-70. [doi:10.1093/clinids/5.1.54]

Wisplinghoff, H., Bischoff, T., Tallent, S.M., et al., 2004. Nosocomial bloodstream infections in US hospitals: analysis of 24179 cases from a prospective nationwide surveillance study. Clin. Infect. Dis., 39(3):309-317. [doi:10.1086/421946]

Ye, J.J., Huang, C.T., Shie, S.S., et al., 2010. Multidrug resistant Acinetobacter baumannii: risk factors for appearance of imipenem resistant strains on patients formerly with susceptible strains. PLoS ONE, 5(4):e9947. [doi:10.1371/journal.pone.0009947]

\section{中文概要}

\section{题 目: 医院获得性血流感染的流行病学和病原学特征分 析: 482 例感染病例的回顾性调查}

目 的: 分析某三级中医院医院获得性血流感染 (nBSIs) 的流行病学和病原学特征, 探索影响 28 天病死 率的相关危险因素。

创新点: 纳入 2009 2011 年所有确诊的血流感染病例, 而 非只依靠血培养结果做判定。收集包括患者的基 础性疾病、侵入性操作等感染相关危险因素、标 本送检及抗菌药物使用情况等全面的临床资料, 分析了 nBSIs 的发生率、病区分布、人群分布、 病原体分布、细菌耐药性及病死率, 并对影响死 亡的危险因素进行了多因素分析。

方 法: 采用回顾性调查的方法, 从病历资料中获得预先 设置好的相关信息。病原体的分离鉴定和药敏检 测由医院微生物实验室专职人员完成。使用 WHONET5.6 软件对药敏结果进行分析。采用 SPSS 16.0 对所有数据进行统计分析, 死亡危险因 素判定先用单因素分析, 再用多因素 Logistic 回 归分析。

结 论: 某三级中医院 nBSIs 的发生率为 5.7/1000 入院人 数, 大肠埃希菌和凝固酶阴性的葡萄球菌 (CoNS) 是检出最多的两种细菌。约有 $1 / 3$ 的肠杆菌对第 三代头狍菌素耐药（表 4），90.7\%的 CoNS 对甲 氧西林耐药（表 3)。碳青需烯类和糖肽类是用 于治疗 nBSIs 使用最广泛的两类抗生素 (图 1)。 感染性休克、血液透析、Pitt 菌血症得分 $>4$ 和尿 路感染是 28 天死亡的危险因素，而合理的经验 性用药可以明显改善预后（表 6）。

关键词: 医院获得性血流感染; 中医院; 流行病学; 病原学 